\title{
Quasi-Exactly Solvable Lie Superalgebras of Differential Operators*
}

\author{
Federico Finkel \\ Artemio González-López \\ Miguel A. Rodríguez \\ Departamento de Física Teórica II \\ Universidad Complutense de Madrid \\ 28040 Madrid, SPAIN
}

February 14, 1997

\begin{abstract}
In this paper, we study Lie superalgebras of $2 \times 2$ matrix-valued first-order differential operators on the complex line. We first completely classify all such superalgebras of finite dimension. Among the finite-dimensional superalgebras whose odd subspace is nontrivial, we find those admitting a finite-dimensional invariant module of smooth vector-valued functions, and classify all the resulting finite-dimensional modules. The latter Lie superalgebras and their modules are the building blocks in the construction of QES quantum mechanical models for spin $1 / 2$ particles in one dimension.
\end{abstract}

PACS numbers: 03.65.Fd, 11.30.Na.

\footnotetext{
*Supported in part by DGICYT Grant PB95-0401.
} 


\section{Introduction}

The discovery of quasi-exactly solvable (QES) spectral problems over the past decade has been a continuous source of interesting mathematical problems. The characteristic feature of a QES Hamiltonian is that a nontrivial portion of its spectrum, but not necessarily all of it, can be computed algebraically. Thus, QES spectral problems occupy an intermediate position between exactly solvable problems, whose spectrum can be completely described, and the vast majority of non-solvable ones. Lie algebras of differential operators have been used extensively to generate physically interesting QES Schrödinger operators, [14], [15], [16]; see also the review book [17]. The basic idea underlying the application of Lie algebras of differential operators to constructing QES models can be summarized as follows: if $\mathfrak{g}$ is a Lie algebra of first-order differential operators with an invariant finite-dimensional $\mathfrak{g}$-module of smooth fuctions $\mathcal{N}$, then any scalar Hamiltonian $H=-\Delta+V$ which can be expressed as a quadratic combination in the generators of $\mathfrak{g}$,

$$
H=\sum_{a, b} c_{a b} T^{a} T^{b}+\sum_{a} c_{a} T^{a}+c_{0}, \quad T^{a} \in \mathfrak{g},
$$

will automatically preserve $\mathcal{N}$. Consequently, if the functions in $\mathcal{N}$ satisfy suitable boundary conditions, one can compute $\operatorname{dim} \mathcal{N}$ eigenfunctions and eigenvalues by diagonalizing the finite matrix which represents $H$ in End $\mathcal{N}$. Therefore, the classification under some well-adapted notion of equivalence of all finite-dimensional Lie algebras of first-order differential operators admitting an invariant module of functions (henceforth called QES Lie algebras) is a good starting point to obtain large families of QES Hamiltonians. In the one-dimensional scalar case the classification is very simple. Indeed, every finite-dimensional QES Lie algebra in one real or complex variable is isomorphic to a subalgebra of (a central extension of) $\mathfrak{s l}_{2}$, [11, [15], [9]. The classification of finite-dimensional QES Lie algebras in two variables is considerably more involved. There are several inequivalent families of QES Lie algebras, some of them of arbitrary dimension, and the real and complex classifications no longer coincide, [6], [8].

The above classification is not, however, the end of the problem. One still has to determine the conditions under which a quadratic combination of the form (1.1) is equivalent to a Schrödinger operator $-\Delta+V$. In the one-dimensional case, it turns out that any quadratic combination (1.1) may be written (locally) in Schrödinger form by the combination of a change of 
the independent variable and a gauge transformation with a non-vanishing function. The situation in higher dimensions is again more complicated. Explicit necessary and sufficient conditions for the equivalence under local diffeomorphisms and gauge transformations of scalar second-order differential operators were first found by É. Cotton, [2]. As a special case, one obtains conditions for the equivalence of a second-order differential operator to a Schrödinger operator acting on a (in general) curved space-time. These conditions have been solved only in some particular cases, and appear to be too complicated to be solved in full generality; see [12] for an in-depth study.

This formalism may be extended to deal with matrix-valued differential equations, suitable for the description of the dynamics of particles with nonzero spin, [14], [1], [3], or the treatment of coupled-channel scattering problems, 18. The procedure for constructing matrix-valued QES Hamiltonians is essentially the same as in the scalar case, with the role of the Lie algebra $\mathfrak{g}$ now being played by a Lie superalgebra $\mathfrak{S}$ of matrix-valued differential operators with an invariant subspace of vector-valued functions, [3]. In principle, $\mathfrak{S}$ need not be finite-dimensional; in practice, however, the only examples constructed so far with this method are associated to finite-dimensional Lie superalgebras, [14, [1], 3]. Lie superalgebras of differential operators are significantly less understood than ordinary Lie algebras. In fact, to the best of the authors' knowledge, no general classification of finite-dimensional Lie superalgebras of differential operators has ever been attempted. The goal of our paper is precisely that of classifying all QES Lie superalgebras of $2 \times 2$ matrix first-order differential operators in one complex variable. The Lie superalgebras thus obtained can be readily used to construct new examples of second-order $2 \times 2$ matrix-valued QES operators, by taking quadratic combinations in the generators of $\mathfrak{S}$ and performing a suitable change of the independent variable and/or a gauge transformation. Necessary and sufficient conditions for the equivalence of a $2 \times 2$ matrixvalued differential operator to a Schrödinger operator were obtained in [3] and [4. A number of QES Lie superalgebras preserving a two-component vector-valued module of polynomials in two complex variables were recently studied in [5].

The paper is organized as follows. In Section 2 we summarize the main results concerning Lie algebras of first-order scalar differential operators on the complex line. In Section 3 we outline our classification scheme for the finitedimensional Lie superalgebras $\mathfrak{S}$ of $2 \times 2$ matrix-valued first-order differential operators. We start with a basic result describing the structure of the even 
and odd subspaces of $\mathfrak{S}$. The classification of all such Lie superalgebras $\mathfrak{S}$ is then shown to be completely equivalent to classifying the finite-dimensional graded subalgebras $\mathfrak{s}$ of the Lie superalgebra $\mathfrak{d}^{1}$ of first-order differential operators in one ordinary variable and one Grassmann variable taking values in the one-generator Grassmann algebra $\Lambda^{1}$. In Section 4 we classify all possible even subalgebras $\mathfrak{l}$ of $\mathfrak{d}^{1}$. The concept of a translation bimodule introduced in this section turns out to play an essential role in the classification, as later shown in Sections 5 and 6. Section 5 is devoted to the classification of the odd subspaces $\mathfrak{s}_{1}$ corresponding to each subalgebra $\mathfrak{l}$ obtained in Section 4 . We first state a necessary condition for $\mathfrak{l}$ to admit a nonzero odd subspace $\mathfrak{s}_{1}$. The list of the even subalgebras $\mathfrak{s}_{0}$ and their corresponding nontrivial odd subspaces $\mathfrak{s}_{1}$ is then presented in Tables 1-4. Finally, in Section 6 the classification is completed by finding all the Lie superalgebras with nontrivial odd subspace that admit a finite-dimensional module $\mathfrak{n} \subset \mathrm{C}^{\infty}\left(\Lambda^{1}\right)$. The associated finite-dimensional modules are also classified, and the results are summarized in Tables 5-9.

\section{Lie algebras of differential operators.}

In this section, we briefly review the basic theory of Lie algebras of first-order scalar differental operators on the complex line, which will serve as a helpful guide in what follows.

Let $\mathcal{D}^{1}$ denote the Lie algebra of differential operators of the form

$$
T=f(z) \partial_{z}+g(z)
$$

where $f$ and $g$ are analytic functions of a complex variable $z$, and the Lie bracket is given by the usual commutator. There are two pseudogroups of transformations acting naturally on $\mathcal{D}^{1}$ which preserve its Lie algebra structure, namely local diffeomorphisms $\bar{z}=\varphi(z)$ and gauge transformations by a non-vanishing function $u(z)$. The action of these transformations on an operator $T$ is given by

$$
T \mapsto \bar{T}, \quad \text { with } \quad \bar{T}(\bar{z})=u(z) \cdot T(z) \cdot u(z)^{-1} .
$$

We shall say that two Lie subalgebras of $\mathcal{D}^{1}$ are equivalent if they can be mapped into each other by a fixed combination of a local diffeomorphism and a gauge transformation. 
The Lie algebra $\mathcal{D}^{1}$ splits naturally into the semi-direct product of the subalgebras of vector fields $\mathcal{V}$ and multiplication operators $\mathcal{M}$ :

$$
\mathcal{D}^{1}=\mathcal{V} \ltimes \mathcal{M}
$$

The classification up to a change of variable of the finite-dimensional subalgebras of $\mathcal{V}$ is due to $\mathrm{S}$. Lie, [10]:

Lemma 2.1 Every nonzero finite-dimensional Lie algebra of vector fields on the line is related by a local change of variable to one of the following Lie algebras:

$$
\mathfrak{h}^{1}=\left\langle\partial_{z}\right\rangle, \quad \mathfrak{h}^{2}=\left\langle\partial_{z}, z \partial_{z}\right\rangle, \quad \mathfrak{h}^{3}=\left\langle\partial_{z}, z \partial_{z}, z^{2} \partial_{z}\right\rangle
$$

Since the natural projection $\pi: \mathcal{D}^{1} \rightarrow \mathcal{V}$ mapping a differential operator (2.1) to its vector field part $f(z) \partial_{z}$ defines a Lie algebra homomorphism, and gauge transformations leave the vector field part unaffected, one may use Lie's classification of vector fields to derive the classification of all finite-dimensional subalgebras of $\mathcal{D}^{1}$, [11], [9]:

Theorem 2.2 Let $\mathfrak{g}$ be a finite-dimensional subalgebra of $\mathcal{D}^{1}$. Then $\mathfrak{g}$ is equivalent to one of the following Lie algebras:

i) $\mathfrak{g}^{0}=\left\langle g_{i}(z) \mid 1 \leq i \leq m\right\rangle$, where the functions $g_{i}$ are linearly independent.

ii) $\mathfrak{g}^{1}=\left\langle\partial_{z}, z^{i} e^{\mu z} \mid 0 \leq i \leq m_{\mu}, \mu \in M\right\rangle$. Here $M$ denotes a finite collection of complex numbers.

iii) $\mathfrak{g}^{2}=\left\langle\partial_{z}, z \partial_{z}, z^{i} \mid 0 \leq i \leq m\right\rangle$.

$\hat{\mathfrak{g}}^{2}=\left\langle\partial_{z}, z \partial_{z}+\alpha\right\rangle$, where $\alpha \in \mathbb{C}$.

iv) $\mathfrak{g}^{3}=\left\langle\partial_{z}, z \partial_{z}, z^{2} \partial_{z}+2 \alpha z, 1\right\rangle$, where $\alpha \in \mathbb{C}$.

$\hat{\mathfrak{g}}^{3}=\left\langle\partial_{z}, z \partial_{z}+\alpha, z^{2} \partial_{z}+2 \alpha z\right\rangle$, where $\alpha \in \mathbb{C}$.

\section{$3 \quad$ Lie superalgebras of differential operators}

We now focus our attention on $2 \times 2$ matrix-valued Lie superalgebras of first-order differential operators. Let $\mathfrak{D}$ denote the associative algebra of all 
$2 \times 2$ matrix differential operators on a complex variable $z$. We introduce a $\mathbb{Z}_{2}$-grading in $\mathfrak{D}$ in the usual way: an operator

$$
T=\left(\begin{array}{ll}
a & b \\
c & d
\end{array}\right)
$$

where $a, b, c$, and $d$ are scalar differential operators, is said to be even if $b=c=0$, and odd if $a=d=0$. This grading, combined with the generalized Lie product

$$
[A, B]_{s}=A B-(-1)^{\operatorname{deg} A \operatorname{deg} B} B A,
$$

endows $\mathfrak{D}$ with a Lie superalgebra structure. We shall be interested in finite-dimensional graded subalgebras of the graded subspace $\mathfrak{D}^{(1)} \subset \mathfrak{D}$ of first-order differential operators. Two such graded subalgebras $\mathfrak{L}$ and $\overline{\mathfrak{L}}$ will be considered equivalent if their elements $T \in \mathfrak{L}$ and $\bar{T} \in \overline{\mathfrak{L}}$ are related by a fixed local change of variable $\bar{z}=\varphi(z)$ and a gauge transformation consistent with the grading:

$$
T \mapsto \bar{T}, \quad \text { with } \quad \bar{T}(\bar{z})=U(z) \cdot T(z) \cdot U(z)^{-1},
$$

where the $2 \times 2$ invertible complex matrix $U(z)$ is either diagonal or antidiagonal. This is a very natural notion of equivalence in the context of QES problems; indeed, if $\mathfrak{N}$ is an $\mathfrak{L}$-module of vector-valued functions and $\overline{\mathfrak{L}}$ is equivalent to $\mathfrak{L}$ under the mapping (3.1), then $\overline{\mathfrak{N}}=U \cdot \mathfrak{N}$ is an invariant module for $\overline{\mathfrak{L}}$. The first aim of this paper consists in classifying under the above equivalence all finite-dimensional graded subalgebras of $\mathfrak{D}$ contained in $\mathfrak{D}^{(1)}$.

We begin with the following elementary result, that we shall state without proof:

Lemma 3.1 Let $\mathfrak{L} \subset \mathfrak{D}^{(1)}$ be a graded subalgebra of $\mathfrak{D}$, and let $\mathfrak{L}_{0}$ and $\mathfrak{L}_{1}$ denote its even and odd subspaces, respectively. We then have:

i) Either all the elements of $\mathfrak{L}_{1}$ are of the form

$$
T_{1}=\left(\begin{array}{cc}
0 & \phi \partial_{z}+\omega \\
\chi & 0
\end{array}\right)
$$

or all its elements are of the form

$$
\tilde{T}_{1}=\left(\begin{array}{cc}
0 & \chi \\
\phi \partial_{z}+\omega & 0
\end{array}\right)
$$

where $\phi, \omega$, and $\chi$ are analytic functions of $z$. 
ii) If $\mathfrak{L}_{1}$ is nonzero, the elements of $\mathfrak{L}_{0}$ are of the form:

$$
T_{0}=\left(\begin{array}{cc}
f \partial_{z}+h_{1} & 0 \\
0 & f \partial_{z}+h_{2}
\end{array}\right),
$$

where $f, h_{1}$, and $h_{2}$ are analytic functions of $z$.

Let us denote by $\mathfrak{D}^{1}$ (respectively $\tilde{\mathfrak{D}}^{1}$ ) the graded subalgebra of $\mathfrak{D}$ generated by all differential operators of the form $T_{0}$ and $T_{1}$ (respectively $T_{0}$ and $\tilde{T}_{1}$ ) in (3.4) and (3.2) (respectively (3.4) and (3.3)). The graded subalgebras $\mathfrak{D}^{1}$ and $\tilde{\mathfrak{D}}^{1}$ are equivalent, since they are related by a gauge transformation with constant matrix

$$
\tilde{U}=\left(\begin{array}{ll}
0 & 1 \\
1 & 0
\end{array}\right) .
$$

Furthermore, the gauge transformations preserving $\mathfrak{D}^{1}$ (or $\tilde{\mathfrak{D}}^{1}$ ) are generated by diagonal matrices $U(z)=\operatorname{diag}(\alpha, \beta)$, where $\alpha$ and $\beta$ are non-vanishing analytic functions of $z$. Therefore, without any loss of generality, we can limit ourselves to classifying the finite-dimensional graded subalgebras of $\mathfrak{D}^{1}$ under local diffeomorphisms and gauge transformations by diagonal matrices.

There is an alternative formalism for describing Lie superalgebras of differential operators which makes use of Grassmann variables. The matrix classification scheme described above is completely equivalent to classifying all finite-dimensional subalgebras $\mathfrak{s}$ of the Lie superalgebra $\mathfrak{d}^{1}$ of first-order differential operators in one ordinary variable $z$ and one Grassmann variable $\theta$ which take values in the one-generator Grassmann algebra $\Lambda^{1}$. The even and odd subspaces of $\mathfrak{d}^{1}$ are generated by differential operators of the form

$$
T_{0}=f(z) \partial_{z}+g(z) \theta \partial_{\theta}+h(z),
$$

and

$$
T_{1}=\theta \phi(z) \partial_{z}+\chi(z) \partial_{\theta}+\omega(z) \theta,
$$

respectively, where $f, g, h, \phi, \chi$, and $\omega$ are analytic functions of $z$. The appropriate equivalence transformations are in this case changes of the independent variables preserving the relation $\left\{\partial_{\theta}, \theta\right\}=1$, namely

$$
\bar{z}=\varphi(z), \quad \bar{\theta}=\beta(z) \theta,
$$


and gauge transformations with a gauge factor of the form $u=\alpha(z)$, where $\alpha$ and $\beta$ are non-vanishing analytic functions of $z$. The standard identifications

$$
\theta \leftrightarrow \sigma^{+}, \quad \partial_{\theta} \leftrightarrow \sigma^{-}, \quad \text { with } \quad \sigma^{+}=\left(\sigma^{-}\right)^{t}=\left(\begin{array}{cc}
0 & 1 \\
0 & 0
\end{array}\right)
$$

lead directly to the equivalence of both formalisms. For the sake of simplicity, we shall use in what follows the Grassmann variable notation. Our first step will thus be to classify all finite-dimensional Lie subalgebras $\mathfrak{l}$ of $\mathfrak{d}_{0}^{1}$, the even subspace of $\mathfrak{d}^{1}$. We will then restrict ourselves to the subalgebras $\mathfrak{s}_{0} \subset \mathfrak{d}_{0}^{1}$ which admit a nontrivial finite-dimensional odd subspace $\mathfrak{s}_{1}$ satisfying the conditions

$$
\left[\mathfrak{s}_{0}, \mathfrak{s}_{1}\right] \subset \mathfrak{s}_{1}, \quad\left\{\mathfrak{s}_{1}, \mathfrak{s}_{1}\right\} \subset \mathfrak{s}_{0}
$$

We will determine all such odd subspaces $\mathfrak{s}_{1}$, and then we will obtain the finite-dimensional modules of functions $\mathfrak{n} \subset \mathrm{C}^{\infty}\left(\Lambda^{1}\right) \simeq \mathrm{C}^{\infty}(\mathbb{C}) \otimes\langle 1, \theta\rangle$ associated to each Lie superalgebra $\mathfrak{s}=\mathfrak{s}_{0} \oplus \mathfrak{s}_{1}$. Note that the $\Lambda^{1}$-valued function $f(z)+g(z) \theta$ is identified with the two-component function $(g(z), f(z))^{t}$ in the matrix formalism.

\section{Lie algebras of even differential operators}

In this section we classify the finite-dimensional subalgebras $\mathfrak{l}$ of the even subspace $\mathfrak{d}_{0}^{1}$ of $\mathfrak{d}^{1}$.

The Lie algebra $\mathfrak{d}_{0}^{1}$ admits the following natural decomposition:

$$
\mathfrak{d}_{0}^{1}=\mathcal{V} \ltimes \mathfrak{a}
$$

where $\mathfrak{a}$ is the abelian Lie algebra of all operators of the form

$$
\hat{T}_{0}=g(z) \theta \partial_{\theta}+h(z) \text {. }
$$

We first observe that the projection $\pi: \mathfrak{d}_{0}^{1} \rightarrow \mathcal{V}$ mapping an even differential operator of the form (3.5) to $f \partial_{z} \in \mathcal{V}$ is a homomorphism of Lie algebras. Moreover, both $\mathfrak{l}$ and its transformed under a change of the odd variable,

$$
\bar{z}=z, \quad \bar{\theta}=\beta(z) \theta,
$$


and/or a gauge transformation clearly have the same projection in $\mathcal{V}$. Therefore, $\pi(\mathfrak{l})$ is either zero or equivalent under a change of the even variable

$$
\bar{z}=\varphi(z), \quad \bar{\theta}=\theta,
$$

to one of the three Lie algebras $\mathfrak{h}^{i}$ in Lemma 2.1. The situation in this respect is completely analogous to the one in the scalar case. Moreover, the Lie algebra $\mathfrak{v}$ of all vector fields of the form

$$
\tilde{T}_{0}=f(z) \partial_{z}+g(z) \theta \partial_{\theta},
$$

is isomorphic to $\mathcal{D}^{1}$, under the identification

$$
\tilde{T}_{0} \mapsto f(z) \partial_{z}+g(z) .
$$

Under this identification, a gauge transformation in $\mathcal{D}^{1}$ by a non-vanishing function $u=\alpha(z)$ becomes a change of the odd variable in $\mathfrak{v}$ of the form $\bar{\theta}=\theta / \alpha$. Thus, the classification of the finite-dimensional subalgebras of $\mathcal{D}^{1}$ in Theorem 2.2 and the classification of the finite-dimensional subalgebras of $\mathfrak{v}$ under changes of variables (3.7) are identical.

We need to introduce some additional notation at this stage. Let $V=$ $\left\langle v_{1}, v_{2}\right\rangle$ be an abstract two-dimensional complex vector space. We define a $V$ translation bimodule as a finite-dimensional $\mathfrak{h}^{1}$-invariant subspace of $\mathcal{M} \otimes V$. The following result provides a detailed description of $V$-translation bimodules:

Proposition 4.1 The most general $V$-translation bimodule is a direct sum

$$
\mathfrak{m}(V, M)=\bigoplus_{\substack{\mu \in M \\ i=1,2,3}} \mathfrak{m}_{\mu}^{i}(V) e^{\mu z},
$$

where $M$ is a finite collection of complex numbers, and

$$
\begin{gathered}
\mathfrak{m}_{\mu}^{1}(V)=\left\langle z^{k} v_{1} \mid 0 \leq k \leq m_{\mu}\right\rangle, \quad \mathfrak{m}_{\mu}^{2}(V)=\left\langle z^{k} v_{2} \mid 0 \leq k \leq n_{\mu}\right\rangle, \\
\mathfrak{m}_{\mu}^{3}(V)=\left\langle z^{m_{\mu}+k} v_{1}+z^{n_{\mu}} \sum_{j=1}^{k} c_{j}^{\mu, k} z^{j} v_{2} \mid 1 \leq k \leq r_{\mu}\right\rangle,
\end{gathered}
$$

with

$$
c_{j}^{\mu, k}=\frac{\left(m_{\mu}+k\right) !\left(n_{\mu}+1\right) !}{\left(n_{\mu}+j\right) !\left(m_{\mu}+k-j+1\right) !} c^{\mu, k-j+1},
$$


where $c^{\mu, 1}, \ldots, c^{\mu, r_{\mu}} \in \mathbb{C}$, and $c^{\mu, 1} \neq 0$. By convention, the indices $m_{\mu}, n_{\mu}$, and $r_{\mu}$ take the values $-1,-1$, and 0 , respectively, when their corresponding modules $\mathfrak{m}_{\mu}^{i}(V)$ are zero.

Remark. The notation $\mathfrak{m}_{\mu}^{i}(V)$ is an abbreviated notation, since the latter sets actually depend on $\mu$ through the parameters $m_{\mu}, n_{\mu}, r_{\mu}$ and $c^{\mu, l}$.

Proof. The only nontrivial point is the structure of the module $\mathfrak{m}_{\mu}^{3}(V)$ of mixed vectors. A mixed vector $g v_{1}+h v_{2}$ may always be chosen to be proportional to

$$
z^{m_{\mu}+k} e^{\mu z} v_{1}+\sum_{\substack{\nu \in N \\ 1 \leq j \leq s_{\nu}}} c_{\nu, j}^{\mu, k} z^{n_{\nu}+j} e^{\nu z} v_{2}
$$

with $k=1, \ldots, r_{\mu}$ and complex numbers $c_{\nu, j}^{\mu, k}$. Acting with $\partial_{z}$ on each of these vectors for $k=1, \ldots, r_{\mu}$, we immediately obtain $c_{\nu, j}^{\mu, k}=\delta_{\mu \nu} c_{j}^{\mu, k}$, with $c_{j}^{\mu, k}$ given by (4.4) for $j \leq k$ and $c_{j}^{\mu, k}=0$ for $j>k$. Q.E.D.

From now on, we shall use the following conventions. The highest value $i_{\max }$ of an index $i$ labeling a collection of operators $\mathcal{S}=\left\{T_{i} \mid i_{\min } \leq i \leq i_{\max }\right\}$ will be set to $i_{\min }-1$ whenever $\mathcal{S}=\emptyset$. The letters $\alpha, \beta, \gamma, \delta$ will denote complex numbers, while the letter $c$ will be reserved for a nonzero complex number. We shall also define the set $\mathfrak{m}_{0}(V)$ by

$$
\mathfrak{m}_{0}(V)=\mathfrak{m}(V,\{0\}), \quad \text { with } \quad c^{0, k}=\delta_{k 1} c .
$$

Explicitly,

$$
\mathfrak{m}_{0}(V)=\left\langle z^{i} v_{1}, z^{j} v_{2}, z^{k}\left(z^{m} v_{1}+c_{k} z^{n} v_{2}\right)\right\rangle,
$$

with $0 \leq i \leq m, 0 \leq j \leq n, 1 \leq k \leq r$, and

$$
c_{k}=\frac{(m+k) !(n+1) !}{(n+k) !(m+1) !} c .
$$

Note that $c_{k}=c$ if $m=n$.

We are now ready to state the classification theorem for the finite-dimensional subalgebras of $\mathfrak{d}_{0}^{1}$. In what follows, a semicolon ";" will be used to separate those generators which characterize a given set from those which may or may not be present, and

$$
\mathfrak{a}_{0}=\left\langle\theta \partial_{\theta}, 1\right\rangle .
$$


Theorem 4.2 Let $\mathfrak{l}$ be a finite-dimensional subalgebra of $\mathfrak{d}_{0}^{1}$. Then $\mathfrak{l}$ is equivalent to one of the following Lie algebras:

i) $\mathfrak{l}^{0}=\left\langle g_{i}(z) \theta \partial_{\theta}+h_{i}(z) \mid 1 \leq i \leq m\right\rangle$, where the operators are linearly independent.

ii) $\mathfrak{l}^{1}=\left\langle\partial_{z} ; \mathfrak{m}\left(\mathfrak{a}_{0}, M\right)\right\rangle$, where $\mathfrak{m}\left(\mathfrak{a}_{0}, M\right)$ is an $\mathfrak{a}_{0}$-translation bimodule.

iii) $\mathfrak{l}^{2}=\left\langle\partial_{z}, z \partial_{z}+\alpha \theta \partial_{\theta}+\beta ; \mathfrak{m}_{0}\left(\mathfrak{a}_{0}\right)\right\rangle$, where $\mathfrak{m}_{0}\left(\mathfrak{a}_{0}\right)$ is given by (4.5). We also have the constraint $m=n$ if $r \geq 0$.

iv) $\mathfrak{I}^{3}=\left\langle\partial_{z}, z \partial_{z}+\alpha \theta \partial_{\theta}+\beta, z^{2} \partial_{z}+2 \alpha z \theta \partial_{\theta}+2 \beta z ; \theta \partial_{\theta}+\gamma, 1\right\rangle$

Remark. Some of the generators may be simplified depending on the presence of the optional generators. For instance, we may take $\alpha=\beta=0$ in $\mathfrak{l}^{2}$ if $m, n \geq 0$, or the second generator of $\mathfrak{l}^{3}$ may be taken as $z \partial_{z}+\beta$ if $\theta \partial_{\theta}+\gamma$ is present.

Proof. Case $i$ ) is obvious. Let us start with $\mathfrak{l}^{1}$. In view of the isomorphism $\mathfrak{a} \simeq \mathcal{M} \otimes \mathfrak{a}_{0}$, we have:

$$
\mathfrak{l}^{1}=\left\langle\partial_{z}+g \theta \partial_{\theta}+h, \mathfrak{m}\left(\mathfrak{a}_{0}, M\right)\right\rangle,
$$

where $g$ and $h$ may be eliminated by an appropriate change of the odd variable (4.1) and a gauge transformation. Consider now $\mathfrak{l}^{2}$. Commuting $z \partial_{z}+g \theta \partial_{\theta}+h$ with the elements of $\mathfrak{m}\left(\mathfrak{a}_{0}, M\right)$ we conclude that $M=\{0\}$. If there are mixed operators present, that is, if $r=r_{0} \neq 0$ in (4.3), then $m=n$ and $c_{j}^{0, k}=\delta_{k j} c$. Furthermore, we may eliminate $g$ (respectively $h$ ) from $z \partial_{z}$ by a suitable change of the odd variable (respectively gauge transformation) unless it is a constant. Finally, we may also have a generator $z^{2} \partial_{z}+g \theta \partial_{\theta}+h$. Commuting it with the generators in $\mathfrak{m}_{0}\left(\mathfrak{a}_{0}\right)$ we conclude that $\mathfrak{l}^{3} \cap \mathfrak{a}$ is a subspace of $\left\langle\theta \partial_{\theta}+\gamma, 1\right\rangle$. Since

$$
\left[\partial_{z}, z^{2} \partial_{z}+g \theta \partial_{\theta}+h\right]=2 z \partial_{z}+g^{\prime} \theta \partial_{\theta}+h^{\prime},
$$

we conclude that $g=2 \alpha z \theta \partial_{\theta}$ and $h=2 \beta z$, for $\alpha, \beta \in \mathbb{C}$. Q.E.D.

\section{The odd subspaces}

We will determine next the possible finite-dimensional odd subspaces $\mathfrak{s}_{1}$ for each of the families of even Lie algebras $\mathfrak{l}^{i}$ of Theorem 4.2. These odd subspaces must verify the commutation relations (3.8). Let us remark that we 
are left with very few equivalence transformations preserving the canonical forms $\mathfrak{l}^{i}$ listed in Theorem 4.2 to simplify the odd subspaces. In spite of this, we shall see that some of the Lie algebras of Theorem 4.2 admit only trivial finite-dimensional odd subspaces.

Lemma 5.1 Let $\mathfrak{s}_{0}$ be a subalgebra of $\mathfrak{d}_{0}^{1}$, and assume that $\mathfrak{s}_{1}$ is a nontrivial finite-dimensional odd subspace for $\mathfrak{s}_{0}$. If $g(z) \theta \partial_{\theta}+h(z)$ belongs to $\mathfrak{s}_{0}$, then $g(z)$ must be a constant.

We thus have:

Corollary 5.2 Let $\mathfrak{s}$ be a finite-dimensional graded subalgebra of $\mathfrak{d}^{1}$ with a nontrivial odd subspace $\mathfrak{s}_{1}$. The even subalgebra $\mathfrak{s}_{0}$ of $\mathfrak{s}$ is then equivalent to one of the following Lie algebras:

i) $\mathfrak{s}_{0}^{0}=\left\langle\epsilon \theta \partial_{\theta}+h_{1}(z) ; h_{l}(z) \mid 2 \leq l \leq s\right\rangle$, where the functions $h_{l}$ are linearly independent.

ii) $\mathfrak{s}_{0}^{1}=\left\langle\partial_{z} ; \epsilon \theta \partial_{\theta}+\alpha z^{s_{0}+1}, z^{l} e^{\sigma z} \mid 0 \leq l \leq s_{\sigma}, \sigma \in \Sigma\right\rangle$. Here $\Sigma$ is a finite collection of complex numbers, $s_{0}=-1$ if $0 \notin \Sigma$, and $\alpha=0$ if $\epsilon=0$.

iii) $\mathfrak{s}_{0}^{2}=\left\langle\partial_{z}, z \partial_{z}+\alpha \theta \partial_{\theta}+\beta ; \epsilon \theta \partial_{\theta}+\gamma, z^{l} \mid 0 \leq l \leq s\right\rangle$.

iv) $\mathfrak{s}_{0}^{3}=\left\langle\partial_{z}, z \partial_{z}+\alpha \theta \partial_{\theta}+\beta, z^{2} \partial_{z}+2 \alpha z \theta \partial_{\theta}+2 \beta z ; \epsilon \theta \partial_{\theta}+\gamma, 1\right\rangle$.

The parameter $\epsilon$ takes values 0,1 .

In Tables 1-4, we present the possible odd subspaces corresponding to each family of Lie algebras $\mathfrak{s}_{0}^{i}$ in Corollary 5.2. It is convenient at this stage to introduce the following convention: the parameters $\epsilon, \hat{\epsilon}$ and $\tilde{\epsilon}$ will take the values 0,1 , and $\epsilon^{*}=1-\epsilon, \hat{\epsilon}^{*}=1-\hat{\epsilon}$.

As an illustration of how these tables were constructed, we shall examine the Lie superalgebras of types $1_{3}$ and $1_{4}$ in Table 2 . Let $T_{1} \in \mathfrak{s}_{1}$ be an operator of the form (3.6), with $\phi \neq 0$. If $\chi=0$ for all such operators, then another operator $\tilde{T}_{1}=\tilde{\chi} \partial_{\theta}+\tilde{\omega} \theta$, with $\tilde{\chi} \neq 0$, must be present in $\mathfrak{s}_{1}$. Let us assume that $\epsilon=\alpha=0$ in $\mathfrak{s}_{0}^{1}$, and $\chi \neq 0$. Anticommuting $T_{1}$ with itself we conclude that $\phi \chi$ is a constant and $\phi \chi^{\prime}=0$. Thus $T_{1}=\theta \partial_{z}+c \partial_{\theta}+\omega \theta$ for some constant $c \neq 0$. Since $\left[\partial_{z}, T_{1}\right]=\omega^{\prime} \theta$, we conclude that $\omega^{\prime} \in\left\langle z^{i} e^{\mu z}\right| 0 \leq i \leq$ $\left.m_{\mu}, \mu \in M\right\rangle$. Therefore, $\omega=\delta z^{m_{0}+1}$ for some $\delta \in \mathbb{C}$. Computing $\left[z^{l} e^{\sigma z}, T_{1}\right]$ we conclude that $m_{\sigma} \geq s_{\sigma}$ for $0 \neq \sigma \in \Sigma$ and $m_{0} \geq s_{0}-1$. Conversely, from $\left\{T_{1}, z^{i} e^{\mu z} \theta\right\}$ we deduce that $s_{\mu} \geq m_{\mu}$ for $0 \neq \mu \in M$ and $s_{0} \geq m_{0}+1$ (or 
$s_{0} \geq m_{0}$ if $\left.\delta=0\right)$. In any case, we can gauge away the term $\delta z^{m_{0}+1}$ in $T_{1}$ and rescale $\theta$ so that $c=1$ without affecting $\mathfrak{s}_{0}^{1}$. We obtain the Lie superalgebra $1_{4}$ in Table 2 . The choice $\chi=0$ in $T_{1}$ leads to the Case $1_{3}$.

\section{The QES Lie superalgebras}

In this section we will determine which of the Lie superalgebras $\mathfrak{s}$ obtained in Section 5 (whose odd subspace $\mathfrak{s}_{1}$ is nonzero) are QES, i.e. admit a nontrivial finite-dimensional module $\mathfrak{n} \subset \mathrm{C}^{\infty}\left(\Lambda^{1}\right)$, and will classify all such modules. We start with the following elementary result:

Lemma 6.1 Let $\mathfrak{s}$ be a finite-dimensional graded subalgebra of $\mathfrak{d}^{1}$ with a nontrivial odd subspace $\mathfrak{s}_{1}$. Then $\mathfrak{s}$ admits a non-zero finite-dimensional module of functions $\mathfrak{n} \subset \mathrm{C}^{\infty}\left(\Lambda^{1}\right)$ if and only if $\mathfrak{s}_{0} \cap \mathfrak{a} \subset \mathfrak{a}_{0}$.

We shall denote the most general $\langle 1, \theta\rangle$-translation bimodule by

$$
\mathfrak{n}(N)=\bigoplus_{\substack{\nu \in N \\ i=1,2,3}} \mathfrak{n}_{\nu}^{i} e^{\nu z}
$$

where $N$ is a finite collection of complex numbers, and

$$
\begin{gathered}
\mathfrak{n}_{\nu}^{1}=\left\langle z^{k} \mid 0 \leq k \leq p_{\nu}\right\rangle, \quad \mathfrak{n}_{\nu}^{2}=\left\langle z^{k} \theta \mid 0 \leq k \leq q_{\nu}\right\rangle, \\
\mathfrak{n}_{\nu}^{3}=\left\langle z^{p_{\nu}+k}+z^{q_{\nu}} \sum_{j=1}^{k} c_{j}^{\nu, k} z^{j} \theta \mid 1 \leq k \leq t_{\nu}\right\rangle .
\end{gathered}
$$

As in Section 4,

$$
c_{j}^{\nu, k}=\frac{\left(p_{\nu}+k\right) !\left(q_{\nu}+1\right) !}{\left(q_{\nu}+j\right) !\left(p_{\nu}+k-j+1\right) !} c^{\nu, k-j+1},
$$

where $c^{\nu, 1}, \ldots, c^{\nu, t_{\nu}} \in \mathbb{C}$, and $c^{\nu, 1} \neq 0$. We shall also consider the set

$$
\mathfrak{n}_{0}=\mathfrak{n}(\{0\}), \quad \text { with } \quad c^{0, k}=\delta_{k 1} c .
$$

In Table 5, we present the list of the even QES subalgebras $\mathfrak{s}_{0}^{i}$ in Corollary 5.2. The calculations needed to complete Table 5 present no difficulties. In Tables 6-9, we present the possible odd subspaces $\mathfrak{s}_{1}$ corresponding to the even QES subalgebras in Table 5, along with the invariant modules of 
functions for $\mathfrak{s}=\mathfrak{s}_{0} \oplus \mathfrak{s}_{1}$. The image of a module $\mathfrak{n}$ under the action of the elements of $\mathfrak{s}_{1}$ will be denoted by $\mathfrak{s}_{1}[\mathfrak{n}]$.

The case $1_{4}$ in Table 7 perhaps deserves some special attention. According to Table 5, we have $\Sigma=\emptyset,\{1\}$. It follows from Table 2 that $\mathfrak{s}_{1}=\left\langle\theta \partial_{z}+\partial_{\theta} ; \theta\right\rangle$, and $\theta \in \mathfrak{s}_{1} \Rightarrow 1 \in \mathfrak{s}_{0}^{1}$. Assume first that $\theta \notin \mathfrak{s}_{1}$. It is easy to see that $p_{\nu}=q_{\nu}$ in equation (6.1) for $\nu \neq 0$. Remarkably, for $\nu \neq 0$ the parameters $c^{\nu, l}$, $l=1, \ldots, t_{\nu}$, defining the mixed functions in $\mathfrak{n}_{\nu}^{3}$ are no longer arbitrary but of the form

$$
c^{\nu, l}=\frac{\left(q_{\nu}+l\right) !}{\left(q_{\nu}+1\right) !} \nu^{\frac{3}{2}-l} a_{l},
$$

where $a_{l}$ are fixed constants that can be determined recursively from the condition $\left(\theta \partial_{z}+\partial_{\theta}\right)\left[\mathfrak{n}_{\nu}^{3}\right] \subset \mathfrak{n}_{\nu}^{3} / \mathfrak{n}_{\nu}^{2}$. The first ten constants are

$$
a_{l}=1, \frac{1}{2}, \frac{1}{2^{3}}, \frac{1}{2^{4}}, \frac{5}{2^{7}}, \frac{7}{2^{8}}, \frac{3 \cdot 7}{2^{10}}, \frac{3 \cdot 11}{2^{11}}, \frac{3 \cdot 11 \cdot 13}{2^{15}}, \frac{5 \cdot 11 \cdot 13}{2^{16}}, \ldots
$$

Finally, it is easy to see that $p_{0}=q_{0}, q_{0}+1$ and $t_{0}=0$. If $\theta \in \mathfrak{s}_{1}$, then $1 \in \mathfrak{s}_{0}^{1}$ and the associated module is $\mathfrak{n}^{1.1}$, with the constraint $p_{\nu}=q_{\nu}$ for all $\nu \in N$.

The parameters $\alpha$ and $\beta$ appearing in the superalgebras of type $\mathfrak{s}^{3}$ are quantized. This phenomenon, the quantization of the cohomology, has been noticed before in the case of finite-dimensional QES Lie algebras and has received some study, [7], [13]. The parameter $\alpha$ is quantized for all finitedimensional Lie superalgebras of the type $\mathfrak{s}^{3}$, whereas $\beta$ is only quantized in every case after imposing the QES condition. Finally, it is also worth mentioning that the Lie superalgebra $\mathfrak{o s p}(2,2) \simeq \mathfrak{s l}(2 \mid 1)$, extensively used in the construction of QES matrix Hamiltonians (see for instance [14], [1], [3]), is the QES superalgebra $3_{5}$ in Tables 4 and 9 with $\epsilon=1$.

\section{References}

[1] Y. Brihaye and P. Kosinski, Quasi exactly solvable $2 \times 2$ matrix equations, J. Math. Phys. 35 (1994), 3089-3098.

[2] É. Cotton, Sur les invariants différentiels de quelques équations aux dérivées partielles du second ordre, Ann. École Normale 17, (1900) 211244.

[3] F. Finkel, A. González-López, and M.A. Rodríguez, Quasi-exactly solvable spin 1/2 Schrödinger operators, J. Math. Phys., in press. 
[4] F. Finkel and N. Kamran, On the equivalence of matrix valued differential operators to Schrödinger form, J. Nonlin. Math. Phys., in press.

[5] F. Finkel and N. Kamran, The Lie algebraic structure of differential operators admitting invariant spaces of polynomials, preprint q-alg/9612027.

[6] A. González-López, N. Kamran, and P.J. Olver, Quasi-exactly solvable Lie algebras of first order differential operators in two complex variables, J. Phys. A24 (1991), 3995-4008.

[7] A. González-López, J. Hurtubise, N. Kamran, and P.J. Olver, Quantification de la cohomologie des algèbres de Lie de champs de vecteurs et fibrés en droites sur des surfaces complexes compactes, C.R. Acad. Sci. (Paris), Série I, 316 (1993) 1307-1312.

[8] A. González-López, N. Kamran, and P.J. Olver, Real Lie algebras of differential operators and quasi-exactly solvable potentials, Phil. Trans. R. Soc. Lond. A 354 (1996), 1165-1193.

[9] N. Kamran and P.J. Olver, Lie algebras of differential operators and Lie-algebraic potentials, J. Math. Anal. Appl. 145 (1990), 342-356.

[10] S. Lie, Theorie der Transformationsgruppen, Math. Ann. 16 (1880), 441-528.

[11] W. Miller, Jr., Lie Theory and Special Functions, Academic Press, New York, 1968.

[12] R. Milson, On the construction of Q.E.S. Schrödinger operators on homogeneous spaces, J. Math. Phys. 36 (1995), 6004-6027.

[13] G. Post, and N. Van den Hijligenberg, $g l(\lambda)$ and differential operators preserving polynomials, Acta Appl. Math. 44 (1996), 257-268.

[14] M.A. Shifman and A.V. Turbiner, Quantal problems with partial algebraization of the spectrum, Commun. Math. Phys. 126 (1989), 347-365.

[15] A.V. Turbiner, Quasi-exactly solvable problems and $\mathfrak{s l}(2)$ algebra, Commun. Math. Phys. 118 (1988), 467-474. 
[16] A.G. Ushveridze, Quasi-exactly solvable models in quantum mechanics, Sov. J. Part. Nucl. 20 (1989), 504-528.

[17] A.G. Ushveridze, Quasi-exactly solvable models in quantum mechanics, Bristol IOP, 1994.

[18] C. Zhu, Exact analytical solution for coupled time independent Schrödinger equations with certain model potentials, J. Phys. A29 (1996), 1293-1303. 
Table 1: Odd subspaces for Lie algebras of type $\mathfrak{s}_{0}^{0}$.

\begin{tabular}{c|c|c} 
Label & \multicolumn{1}{|c}{$\mathfrak{s}_{1}$} & \multicolumn{1}{|c}{ Rules } \\
\hline \hline $0_{1}$ & $\left\langle\theta\left(\phi_{i} \partial_{z}+\omega_{i}\right), h_{l}^{\prime} \phi_{i} \theta\right\rangle$ & $1 \leq i \leq m$. \\
\hline $0_{2}$ & $\left\langle\chi_{i} \partial_{\theta}+\omega_{i} \theta\right\rangle$ & $1 \leq i \leq m ; \quad \chi_{i} \omega_{i}=0$ if $\epsilon=1 ;$ \\
& & $\chi_{i} \omega_{k}+\chi_{k} \omega_{i} \in \mathfrak{s}_{0}^{0}$.
\end{tabular}


Table 2: Odd subspaces for Lie algebras of type $\mathfrak{s}_{0}^{1}$.

\begin{tabular}{|c|c|c|}
\hline Label & $\mathfrak{s}_{1}$ & Rules \\
\hline $1_{1}$ & $\mathfrak{m}\left(\left\langle\theta \partial_{z}, \theta\right\rangle, M\right)$ & $\begin{array}{l}n_{\mu+\sigma} \geq s_{\sigma}+m_{\mu}+r_{\mu}-\delta_{\alpha 0} \delta_{\sigma 0} \quad \text { if } \\
\text { i) } m_{\mu} \geq 0 \text { or } r_{\mu} \geq 1 \\
\text { ii) } \sigma \in \Sigma, \text { with } \alpha \neq 0 \text { if } s_{0}=0 .\end{array}$ \\
\hline $1_{2}$ & $\mathfrak{m}\left(\left\langle\partial_{\theta}, \theta\right\rangle, M\right)$ & $\begin{array}{l}r_{\mu}=0 \text { if } \epsilon=1 . \\
m_{\mu}, n_{\tilde{\mu}} \geq 0 \Rightarrow s_{\mu+\tilde{\mu}} \geq m_{\mu}+n_{\tilde{\mu}} . \\
r_{\mu} \geq 1, n_{\tilde{\mu}} \geq 0 \Rightarrow s_{\mu+\tilde{\mu}} \geq m_{\mu}+r_{\mu}+n_{\tilde{\mu}} . \\
r_{\mu} \geq 1, m_{\tilde{\mu}} \geq 0 \Rightarrow s_{\mu+\tilde{\mu}} \geq n_{\mu}+r_{\mu}+m_{\tilde{\mu}} . \\
r_{\mu}, r_{\tilde{\mu}} \geq 1, m_{\mu}+n_{\tilde{\mu}} \neq m_{\tilde{\mu}}+n_{\mu} \\
\quad \Rightarrow s_{\mu+\tilde{\mu}} \geq r_{\mu}+r_{\tilde{\mu}}+\max \left(m_{\mu}+n_{\tilde{\mu}}, m_{\tilde{\mu}}+n_{\mu}\right) . \\
\quad r_{\mu}, r_{\tilde{\mu}} \geq 1, m_{\mu}+n_{\tilde{\mu}}=m_{\tilde{\mu}}+n_{\mu}, S_{\mu \tilde{\mu}}^{p} \neq \emptyset \text { for some } p \\
\quad \Rightarrow s_{\mu+\tilde{\mu}} \geq m_{\mu}+n_{\tilde{\mu}}+\max _{S_{\mu \tilde{\mu}}^{p} \neq \emptyset} p .\end{array}$ \\
\hline $1_{3}$ & $\left\langle\theta \partial_{z}, \partial_{\theta}+\hat{\epsilon} z^{m_{0}+1} \theta ; z^{i} e^{\mu z} \theta\right\rangle$ & $\begin{array}{l}\hat{\epsilon}=1 \Rightarrow \epsilon=\alpha=0 \\
\Sigma^{*}=M^{*}, \quad s_{\mu}=m_{\mu} \text {. Either } \\
\text { i) } \hat{\epsilon}=\alpha=0 \Rightarrow s_{0}=m_{0}, m_{0}+1 \\
\text { ii) } \hat{\epsilon}=0, \alpha \neq 0 \Rightarrow s_{0}=m_{0} \\
\text { iii) } \hat{\epsilon}=1 \Rightarrow s_{0}=m_{0}+1\end{array}$ \\
\hline $1_{4}$ & $\left\langle\theta \partial_{z}+\partial_{\theta} ; z^{i} e^{\mu z} \theta\right\rangle$ & $\begin{array}{l}\epsilon=\alpha=0 \\
\Sigma^{*}=M^{*}, \quad s_{\mu}=m_{\mu}, \quad s_{0}=m_{0}, m_{0}+1\end{array}$ \\
\hline
\end{tabular}

The set $S_{\mu \tilde{\mu}}^{p} \subset \mathbb{Z}^{4}$ in Case $1_{2}$ is defined as

$S_{\mu \tilde{\mu}}^{p}=\left\{(k, \tilde{k}, j, \tilde{\jmath}) \mid k+\tilde{\jmath}=\tilde{k}+j=p\right.$ and $\left.c_{j}^{\mu, k}+c_{\tilde{\jmath}}^{\tilde{\mu}, \tilde{k}} \neq 0 ; 1 \leq j \leq k \leq r_{\mu}, 1 \leq \tilde{\jmath} \leq \tilde{k} \leq r_{\tilde{\mu}}\right\}$

In Cases $1_{3}$ and $1_{4}$ the index $i=0, \ldots, m_{\mu}, \mu \in M$. 
Table 3: Odd subspaces for Lie algebras of type $\mathfrak{s}_{0}^{2}$.

\begin{tabular}{|c|c|c|}
\hline Label & $\mathfrak{s}_{1}$ & Rules \\
\hline $22_{1}$ & $\mathfrak{m}_{0}\left(\left\langle\theta \partial_{z}, \theta\right\rangle\right)$ & $\begin{array}{l}r \geq 1 \Rightarrow m=n+1 \text { and } s=-1,0 . \\
r=0, s>0, m \geq 0 \Rightarrow n \geq m+s-1 .\end{array}$ \\
\hline $22_{2}$ & $\mathfrak{m}_{0}\left(\left\langle\partial_{\theta}, \theta\right\rangle\right)$ & $\begin{array}{l}r=0 \text { if } \epsilon=1 . \\
r \geq 1 \Rightarrow m=n+2 \alpha ; \alpha \text { semi-integer } \geq-(n+1) / 2 . \\
s \geq m+n+2 r \text { if } m, n \geq 0 \text { or } r \geq 1 .\end{array}$ \\
\hline 23 & $\left\langle\theta \partial_{z}, \partial_{\theta}+\hat{\epsilon} z^{m+1} \theta ; z^{i} \theta\right\rangle$ & $\begin{array}{l}\hat{\epsilon}=1 \Rightarrow s=m+1=-2 \alpha \text { and } \epsilon=0 \\
\hat{\epsilon}=0 \Rightarrow s=m, m+1\end{array}$ \\
\hline 24 & $\left\langle\theta\left(z \partial_{z}+\delta\right), \theta \partial_{z}, \partial_{\theta}+\epsilon^{*} \hat{\epsilon} \theta ; z^{i} \theta\right\rangle$ & $\begin{array}{l}\alpha=0 \text { and either: } \\
\text { i) } s=m, \delta=\beta \text {, and } \hat{\epsilon}=0 \text {. } \\
\text { ii) } s=m+1=0 \text {. }\end{array}$ \\
\hline 25 & $\left\langle\theta \partial_{z}+\hat{\epsilon}^{*} z \partial_{\theta}, \hat{\epsilon} z \partial_{\theta}, \partial_{\theta} ; z^{i} \theta\right\rangle$ & $\begin{array}{l}s=m+1 \text { and either: } \\
\text { i) } \epsilon=\beta=0, \quad \alpha=1 \\
\text { ii) } \epsilon=\hat{\epsilon}=1, \quad \beta=-\gamma\end{array}$ \\
\hline $2_{6}$ & $\left\langle\theta \partial_{z}+\partial_{\theta} ; z^{i} \theta\right\rangle$ & $\epsilon=0, \alpha=\frac{1}{2}, s=m, m+1$ \\
\hline 27 & $\left\langle\theta z \partial_{z}+\partial_{\theta}+\delta \theta, \theta \partial_{z} ; z^{i} \theta\right\rangle$ & $\begin{array}{l}\epsilon=\alpha=0 \text { and either: } \\
\text { i) } s=m, \delta=\beta . \\
\text { ii) } s=m+1=0 .\end{array}$ \\
\hline
\end{tabular}

In Cases $2_{3}-2_{7}$ the index $i=0, \ldots, m$. 
Table 4: Odd subspaces for Lie algebras of type $\mathfrak{s}_{0}^{3}$.

\begin{tabular}{c|l|l} 
Label & \multicolumn{1}{|c|}{$\mathfrak{s}_{1}$} & \multicolumn{1}{|c}{ Rules } \\
\hline \hline $3_{1}$ & $\left\langle\theta z^{i-1}\left(z \partial_{z}+2 \beta i / m\right)\right\rangle$ & $\alpha=1-\frac{m}{2}$, and $\beta=0$ if $m=0$. \\
\hline $3_{2}$ & $\left\langle z^{i} \theta ; \theta z^{m+1}\left(z \partial_{z}+2 \beta\right), \theta z^{j} \partial_{z}\right\rangle$ & $2 \alpha=-m ; j=0, \ldots, m+1$. \\
\hline $3_{3}$ & $\left\langle z^{i} \partial_{\theta}\right\rangle$ & $2 \alpha=m$. \\
\hline $3_{4}$ & $\left\langle\partial_{\theta}+\epsilon^{*} \hat{\epsilon} \theta ; \hat{\epsilon} \theta, \theta z^{j-1}\left(z \partial_{z}+j \beta\right)\right\rangle$ & $\alpha=0 ; j=0,1,2$. \\
& & $1 \in \mathfrak{s}_{0}^{3}$ if $\hat{\epsilon}=1$. \\
\hline $3_{5}$ & $\left\langle\theta z \partial_{z}+\epsilon^{*} z \partial_{\theta}+2 \beta \theta, \theta \partial_{z}+\epsilon^{*} \partial_{\theta}, \epsilon z \partial_{\theta}, \epsilon \partial_{\theta}\right\rangle$ & $2 \alpha=1 ; \gamma=-2 \beta$ if $\epsilon=1$. \\
\hline $3_{6}$ & $\left\langle\theta \partial_{z}, z^{j} \partial_{\theta}\right\rangle$ & $\alpha=1 ; \beta=0 ; j=0,1,2$.
\end{tabular}

The index $i$ takes values $i=0, \ldots, m$. 
Table 5: Even QES subalgebras $\mathfrak{s}_{0}$ and associated modules $\mathfrak{n}$.

\begin{tabular}{l|l|l}
\multicolumn{1}{c|}{$\mathfrak{s}_{0}$} & \multicolumn{1}{|c}{$\mathfrak{n}$} & \multicolumn{1}{c}{ Rules } \\
\hline \hline $\mathfrak{s}_{0}^{0}=\left\langle\epsilon \theta \partial_{\theta}+\alpha ; 1\right\rangle$ & $\mathfrak{n}^{0.0}=\left\langle f_{j}+g_{j} \theta\right\rangle$ & $\epsilon=\alpha=0$. \\
\cline { 2 - 3 } & $\mathfrak{n}^{0.1}=\left\langle f_{j}, g_{k} \theta\right\rangle$ & $\epsilon=1$. \\
\hline \multirow{2}{*}{$\mathfrak{s}_{0}^{1}=\left\langle\partial_{z}, \epsilon \theta \partial_{\theta}+\alpha ; 1\right\rangle$} & $\mathfrak{n}^{1.0}=\mathfrak{n}(N)$ & $\epsilon=\alpha=0$. \\
\cline { 2 - 3 } & $\mathfrak{n}^{1.1}=\bigoplus_{\nu \in N} \mathfrak{n}_{\nu}^{1} \oplus \mathfrak{n}_{\nu}^{2}$ & $\epsilon=1$. \\
\hline $\mathfrak{s}_{0}^{2}=\left\langle\partial_{z}, z \partial_{z}+\alpha \theta \partial_{\theta}+\beta ; \epsilon \theta \partial_{\theta}+\gamma, 1\right\rangle$ & $\mathfrak{n}^{2.0}=\mathfrak{n}_{0}$ & $\epsilon=0 ; t \geq 1 \Rightarrow \alpha=p-q$. \\
\cline { 2 - 3 } & $\mathfrak{n}^{2.1}=\left\langle z^{j}, z^{k} \theta\right\rangle$ & $\epsilon=1$. \\
\hline $\mathfrak{s}_{0}^{3}=\left\langle\partial_{z}, z \partial_{z}+\alpha \theta \partial_{\theta}+\beta, z^{2} \partial_{z}\right.$ & $\mathfrak{n}^{3.0}=\left\langle z^{j}(1+c \theta)\right\rangle$ & $\epsilon=\alpha=0 ; 2 \beta=-p$. \\
\cline { 2 - 3 }$\left.+2 \alpha z \theta \partial_{\theta}+2 \beta z ; \epsilon \theta \partial_{\theta}+\gamma, 1\right\rangle$ & $\mathfrak{n}^{3.1}=\left\langle z^{j}\right\rangle$ & $2 \beta=-p$. \\
\cline { 2 - 3 } & $\mathfrak{n}^{3.2}=\left\langle z^{k} \theta\right\rangle$ & $2(\alpha+\beta)=-q$. \\
\cline { 2 - 3 } & $\mathfrak{n}^{3.3}=\left\langle z^{j}, z^{k} \theta\right\rangle$ & $2 \beta=-p ; 2(\alpha+\beta)=-q$.
\end{tabular}

The indices $j, k$ take values $j=0, \ldots, p, k=0, \ldots, q$

Table 6: Odd subspaces $\mathfrak{s}_{1}$ for even QES Lie algebras $\mathfrak{s}_{0}^{0}$ and $\mathfrak{s}$-modules $\mathfrak{n}$.

\begin{tabular}{c|l|l|l} 
Label & \multicolumn{1}{|c|}{$\mathfrak{s}_{1}$} & \multicolumn{1}{c|}{$\mathfrak{n}$} & Rules \\
\hline \hline $0_{1}$ & $\left\langle\theta\left(\phi_{i} \partial_{z}+\omega_{i}\right)\right\rangle$ & $\mathfrak{n}^{0 . \epsilon}+\mathfrak{s}_{1}\left[\mathfrak{n}^{0 . \epsilon}\right]$ & \\
\hline $0_{2 a}$ & $\left\langle\chi_{i} \partial_{\theta}\right\rangle$ & $\mathfrak{n}^{0 . \epsilon}+\mathfrak{s}_{1}\left[\mathfrak{n}^{0 . \epsilon}\right]$ & \\
\hline $0_{2 b}$ & $\left\langle\partial_{\theta}+\hat{\epsilon}^{*} \theta ; \hat{\epsilon} \theta\right\rangle$ & $\left\langle f_{j}+g_{j} \theta, g_{j}+f_{j} \theta\right\rangle$ & $\hat{\epsilon}=\epsilon=0, \quad 1 \in \mathfrak{s}_{0}^{0}$. \\
\cline { 3 - 4 } & & $\left\langle f_{j}, f_{j} \theta\right\rangle$ & $\hat{\epsilon}=1, \quad 1 \in \mathfrak{s}_{0}^{0}$.
\end{tabular}

The indices $i, j$ take values $i=1, \ldots, m, j=1, \ldots, p$ 
Table 7: Odd subspaces $\mathfrak{s}_{1}$ for even QES Lie algebras $\mathfrak{s}_{0}^{1}$ and $\mathfrak{s}$-modules $\mathfrak{n}$.

\begin{tabular}{|c|c|c|c|}
\hline Label & $\mathfrak{s}_{1}$ & $\mathfrak{n}$ & Rules \\
\hline $1_{1}$ & $\overline{\mathfrak{m}}\left(\left\langle\theta \partial_{z}, \theta\right\rangle, M\right)$ & $\mathfrak{n}^{1 . \epsilon}+\mathfrak{s}_{1}\left[\mathfrak{n}^{1 . \epsilon}\right]$ & \\
\hline $1_{2 a}$ & $\left\langle z^{i} e^{\mu z} \partial_{\theta}\right\rangle$ & $\mathfrak{n}^{1 . \epsilon}+\mathfrak{s}_{1}\left[\mathfrak{n}^{1 . \epsilon}\right]$ & $0 \leq i \leq m_{\mu}, \quad \mu \in M$ \\
\hline $1_{2 b}$ & $\left\langle\partial_{\theta}+\hat{\epsilon} \theta ; \hat{\epsilon}^{*} \theta\right\rangle$ & $\mathfrak{n}^{1 . \hat{\epsilon}^{*}}$ & $\begin{array}{l}1 \in \mathfrak{s}_{0}^{1}, \text { and } \epsilon=1 \Rightarrow \hat{\epsilon}=0 \\
p_{\nu}=q_{\nu} ; \quad c^{\nu, k}=\delta_{k, 1}\end{array}$ \\
\hline $1_{3}$ & $\left\langle\theta \partial_{z}, \partial_{\theta}+\hat{\epsilon} \theta ; \theta\right\rangle$ & $\left\langle\mathfrak{n}^{1.1} ; \tilde{\epsilon} z^{q_{0}+1}(1+\hat{\epsilon} \theta)\right\rangle$ & $\begin{array}{l}\theta \in \mathfrak{s}_{1} \Rightarrow 1 \in \mathfrak{s}_{0}^{1} \text { and } \tilde{\epsilon}=0 \\
\hat{\epsilon}=1 \Rightarrow 1 \in \mathfrak{s}_{0}^{1} \text { and } \epsilon=0 \\
p_{\nu}=q_{\nu}\end{array}$ \\
\hline \multirow[t]{2}{*}{$1_{4}$} & \multirow[t]{2}{*}{$\left\langle\theta \partial_{z}+\partial_{\theta} ; \theta\right\rangle$} & $\mathfrak{n}^{1.0}$ & $\begin{array}{l}\theta \notin \mathfrak{s}_{1} \text {. If } \nu \neq 0, \text { we have: } \\
\text { i) } p_{\nu}=q_{\nu} . \\
\text { ii) } c^{\nu, l}=\frac{\left(q_{\nu}+l\right) !}{\left(q_{\nu}+1\right) !} \nu^{\frac{3}{2}-l} a_{l} \text {, with } \\
\quad a_{l} \text { fixed constants and } l=1, \ldots, t_{\nu} \text {. } \\
p_{0}=q_{0}, q_{0}+1 ; \quad t_{0}=0 \text {. }\end{array}$ \\
\hline & & $\mathfrak{n}^{1.1}$ & $\theta \in \mathfrak{s}_{1}^{1} ; \quad 1 \in \mathfrak{s}_{0}^{1} ; \quad p_{\nu}=q_{\nu}$ \\
\hline
\end{tabular}

The parameter $\epsilon=0$ in Case $1_{4}$. 
Table 8: Odd subspaces $\mathfrak{s}_{1}$ for even QES Lie algebras $\mathfrak{s}_{0}^{2}$ and $\mathfrak{s}$-modules $\mathfrak{n}$.

\begin{tabular}{|c|c|c|c|}
\hline Label & $\mathfrak{s}_{1}$ & $\mathfrak{n}$ & Rules \\
\hline $22_{1}$ & $\overline{\mathfrak{m}_{0}\left(\left\langle\theta \partial_{z}, \theta\right\rangle\right)}$ & $\mathfrak{n}^{2 . \epsilon}+\mathfrak{s}_{1}\left[\mathfrak{n}^{2 . \epsilon}\right]$ & $r \geq 1 \Rightarrow m=n+1$ \\
\hline $22_{2 a}$ & $\left\langle z^{i} \partial_{\theta}\right\rangle$ & $\mathfrak{n}^{2 . \epsilon}+\mathfrak{s}_{1}\left[\mathfrak{n}^{2 . \epsilon}\right]$ & $0 \leq i \leq m$ \\
\hline $22_{2 b}$ & $\left\langle\left\langle\partial_{\theta}+\hat{\epsilon} \theta ; \hat{\epsilon}^{*} \theta\right\rangle\right.$ & $\mathfrak{n}^{2 . \hat{\epsilon}^{*}}$ & $\begin{array}{l}1 \in \mathfrak{s}_{0}^{2}, \text { and } \hat{\epsilon}=1 \Rightarrow \epsilon=\alpha=0 . \\
p=q ; \quad c=1 .\end{array}$ \\
\hline $22_{3}$ & $\left\langle\theta \partial_{z}, \partial_{\theta}+\hat{\epsilon} \theta ; \theta\right\rangle$ & $\left\langle\mathfrak{n}^{2.1} ; \tilde{\epsilon} z^{q+1}(1+\hat{\epsilon} \theta)\right\rangle$ & $\begin{array}{l}\theta \in \mathfrak{s}_{1} \Rightarrow 1 \in \mathfrak{s}_{0}^{2} \text { and } \tilde{\epsilon}=0 \\
\hat{\epsilon}=1 \Rightarrow 1 \in \mathfrak{s}_{0}^{2} \text { and } \epsilon=\alpha=0 \\
p=q\end{array}$ \\
\hline $22_{4}$ & $\left\langle\theta\left(z \partial_{z}+\delta\right), \theta \partial_{z}, \partial_{\theta}+\epsilon^{*} \hat{\epsilon} \theta ; \theta\right\rangle$ & $\left\langle\mathfrak{n}^{2.1} ; \tilde{\epsilon} z^{q+1}\left(1+\epsilon^{*} \hat{\epsilon} \theta\right)\right\rangle$ & $\begin{array}{l}\alpha=0, \quad p=q \\
1 \notin \mathfrak{s}_{0}^{2} \Rightarrow \theta \notin \mathfrak{s}_{1}, \delta=\beta, \hat{\epsilon}=0 \\
\tilde{\epsilon}=1 \Rightarrow \theta \notin \mathfrak{s}_{1}, \delta=-(q+1)\end{array}$ \\
\hline 25 & $\left\langle\theta \partial_{z}+\hat{\epsilon}^{*} z \partial_{\theta}, \hat{\epsilon} z \partial_{\theta}, \partial_{\theta}\right\rangle$ & $\mathfrak{n}^{2.1}$ & $\begin{array}{l}p=q+1 \text { and either: } \\
\text { i) } \epsilon=\beta=0, \quad \alpha=1 \\
\text { ii) } \epsilon=\hat{\epsilon}=1, \beta=-\gamma\end{array}$ \\
\hline $22_{6}$ & $\left\langle\theta \partial_{z}+\partial_{\theta} ; \theta\right\rangle$ & $\mathfrak{n}^{2.1}$ & $\begin{array}{l}\theta \in \mathfrak{s}_{1} \Rightarrow 1 \in \mathfrak{s}_{0}^{2} \text { and } p=q . \\
\theta \notin \mathfrak{s}_{1} \Rightarrow p=q, q+1 .\end{array}$ \\
\hline 27 & $\left\langle\theta z \partial_{z}+\partial_{\theta}+\delta \theta, \theta \partial_{z} ; \theta\right\rangle$ & $\begin{array}{l}\left\langle\mathfrak{n}^{2.1} ; \tilde{\epsilon} z^{q+1}(1\right. \\
\left.\left.\quad+(\delta+q+1)^{\frac{1}{2}} \theta\right)\right\rangle\end{array}$ & $\begin{array}{l}\epsilon=\alpha=0, \quad p=q . \\
1 \notin \mathfrak{s}_{0}^{2} \Rightarrow \theta \notin \mathfrak{s}_{1} \text { and } \delta=\beta . \\
\theta \in \mathfrak{s}_{1} \Rightarrow \tilde{\epsilon}=0 .\end{array}$ \\
\hline
\end{tabular}


Table 9: Odd subspaces $\mathfrak{s}_{1}$ for even QES Lie algebras $\mathfrak{s}_{0}^{3}$ and $\mathfrak{s}$-modules $\mathfrak{n}$.

\begin{tabular}{|c|c|c|c|}
\hline Label & $\mathfrak{s}_{1}$ & $\mathfrak{n}$ & Rules \\
\hline \multirow[t]{4}{*}{$33_{1}$} & \multirow{4}{*}{$\left\langle\theta z^{i-1}\left(z \partial_{z}+2 \beta i / m\right)\right\rangle$} & $\langle\overline{\langle 1+\theta\rangle}$ & $\bar{c} \epsilon=\beta=0, m=2$. \\
\hline & & $\langle 1\rangle$ & $\beta=0$ \\
\hline & & $\mathfrak{n}^{3.2}$ & $m \geq 1 ; 2 \beta=m-q-2$ \\
\hline & & $\mathfrak{n}^{3.3}$ & $m=1,2 ; 2 \beta=m-q-2=-p$ \\
\hline $3_{2}$ & $\left\langle z^{i} \theta ; \theta z^{m+1}\left(z \partial_{z}+2 \beta\right), \theta z^{j} \partial_{z}\right\rangle$ & $\mathfrak{n}^{3.2}, \mathfrak{n}^{3.3}$ & $\begin{array}{l}2 \alpha=-m ; \quad j=0, \ldots, m+1 \\
2 \beta=m-q=-p\end{array}$ \\
\hline $33_{3}$ & $\left\langle z^{i} \partial_{\theta}\right\rangle$ & $\mathfrak{n}^{3.1}, \mathfrak{n}^{3.3}$ & $2 \alpha=m ;-2 \beta=p=m+q$ \\
\hline \multirow[t]{2}{*}{$34 a$} & \multirow[t]{2}{*}{$\left\langle\partial_{\theta} ; \theta, \theta z^{j-1}\left(z \partial_{z}+j \beta\right)\right\rangle$} & $\mathfrak{n}^{3.1}$ & $\theta \notin \mathfrak{s}_{1} ; 2 \beta=-p$ \\
\hline & & $\mathfrak{n}^{3.3}$ & $2 \beta=-p=-q$, and $\theta \in \mathfrak{s}_{1} \Rightarrow 1 \in \mathfrak{s}_{0}^{3}$ \\
\hline \multirow[t]{2}{*}{$3_{4 b}$} & \multirow[t]{2}{*}{$\overline{\left\langle\partial_{\theta}+\theta ; \hat{\epsilon} \theta z^{j-1}\left(z \partial_{z}+j \beta\right)\right\rangle}$} & $\mathfrak{n}^{3.0}$ & $c=1 ; 2 \beta=-\hat{\epsilon}^{*} p$ \\
\hline & & $\mathfrak{n}^{3.3}$ & $2 \beta=-p=-q$ \\
\hline \multirow[t]{2}{*}{$3_{5}$} & \multirow{2}{*}{$\begin{array}{r}\left\langle\theta z \partial_{z}+\epsilon^{*} z \partial_{\theta}+2 \beta \theta,\right. \\
\left.\theta \partial_{z}+\epsilon^{*} \partial_{\theta}, \epsilon z \partial_{\theta}, \epsilon \partial_{\theta}\right\rangle\end{array}$} & $\langle 1\rangle$ & $2 \alpha=1 ; \beta=0$ \\
\hline & & $\mathfrak{n}^{3.3}$ & $2 \alpha=1 ;-2 \beta=p=q+1$ \\
\hline $3_{6}$ & $\left\langle\theta \partial_{z}, z^{j} \partial_{\theta}\right\rangle$ & $\langle 1\rangle$ & $\alpha=1 ; \beta=0 ; j=0,1,2$. \\
\hline
\end{tabular}

The index $i$ takes values $i=0, \ldots, m$. In Case $3_{1}, \alpha=1-\frac{m}{2}$ and $\beta=0$ if $m=0$. In Cases $3_{4}, \alpha=0$ and $j=0,1,2$. In Case $3_{4 b}$, we also have $\epsilon=0$ and $1 \in \mathfrak{s}_{0}^{3}$. In Case $3_{5}$, $\gamma=-2 \beta$ if $\epsilon=1$. 